\title{
Verification of ADS-B positioning by direction of arrival estimation
}

\author{
CHRISTOPH RECK ${ }^{1}$, MAX S. REUTHER ${ }^{1}$, ALEXANDER JASCH ${ }^{2}$ AND LORENZ-PETER SCHMIDT ${ }^{1}$
}

\begin{abstract}
ADS-B provides convenient means of air traffic control (ATC) for its low cost and simple ground station hardware. In a low percentage of cases, aircraft positions transmitted via ADS-B are error prone due to e.g. faulty wiring with onboard navigation systems. In the proposed approach, direction of arrival (DOA) estimation is used to verify ADS-B airborne positions. The potential positioning error of $A D S-B$ is thereby evaluated by comparing DOA estimates to DOA values calculated from $A D S-B$ references and the sensor position and orientation. To prove the accuracy of the applied DOA estimation sensor, an additional measurement campaign using a dedicated measurement aircraft has been conducted.
\end{abstract}

Keywords: Radar applications, Smart antennas, Direction of arrival estimation

Received 15 November 2011; Revised 18 January 2012; first published online 22 February 2012

\section{INTRODUCTION}

During the last few decades a significant increase in air traffic is recognized. The high traffic load needs to be carefully coordinated by air traffic control (ATC) to satisfy rigorous security demands.

To provide high-quality ATC, its operators are depending on information gathered by radar sensors. Classic primary surveillance radar (PSR) approaches require a large number of expensive and energy consuming ground stations. In order to cut down the number of primary radar stations, the nondependent use of secondary surveillance radar (SSR) transponders for aircraft positioning is evaluated by ATC organizations.

The automatic-dependent surveillance broadcast (ADS-B) is based on the SSR Mode S protocol. Unlike a regular SSR system, which broadcasts radio telegrams mainly on prior request by ground stations, ADS-B uses spontaneous transponder broadcasts triggered randomly based on the Aloha-Protocol. Offering more information than just altitude and identification, ADS-B also transmits the carrying aircraft's position as it is gathered by its onboard navigation system.

Additionally, ground speed, heading, and many other information are provided. As the number of aircraft equipped with ADS-B is rising (currently $65 \%$ of Mode $S$ equipped aircraft $[1])$, the system becomes increasingly attractive to feed ATC displays.

According to field studies [2], the most part of ADS-B transponders are broadcasting reliable positioning information, where positions' root mean-squared error (RMSE)

${ }^{1}$ Chair for High Frequency Technology, FAU Erlangen-Nuremberg, Cauerstrasse 9, 91058 Erlangen, Germany. Phone: +49 91318525472

${ }^{2}$ Institute of Flight Guidance, TU Braunschweig, Hermann-Blenk-Str. 27, 38108 Braunschweig, Germany

Corresponding author:

C. Reck

Email: christoph@lhft.eei.uni-erlangen.de is following a Rayleigh distribution with a mean value of around 250. This would on the one hand mean an acceptable error for monitoring en route traffic. On the other hand, there are some transponders that produce much larger errors. One possible problem leading to those large errors is improper wiring of the ADS-B transponder with the onboard navigation system or inertial measurement unit (IMU).

This is one of the reasons why a standalone ADS-B solution without ground-based validation techniques is not considered reliable enough for ATC. Nevertheless, due to its immense cost the installation of PSR, SSR or multilateration is sometimes not economically justifiable. As shown in [3], the risk of relying on a pure ADS-B system is taken in such a case by NAV CANADA at Hudson Bay.

The situation changes if the ADS-B ground station has the capability to cross-check the transmitted position with an independent measurement as shown in Fig. 1(a). By using ADS-B ground stations with direction of arrival estimation capability, verification of ADS-B transmitted positions can either be done by a single or better by two linked ground stations.

In our previous work $[4,5]$, subspace-based DOA estimation by multichannel receivers showed reliable estimates. In this contribution, the possible interaction of such a DOA sensor with an included ADS-B receiver is discussed. Therefore results gathered by field tests using ADS-B as well as by a dedicated measurement aircraft are compared. Finally, possible applications of such sensors at an existing ADS-B system as shown in [3] are discussed.

\section{DIRECTIDN DF ARRIVAL ESTIMATION}

The principle of DOA estimation can be visualized by assuming a plane wave (far-field assumption) impinging on an array of antennas as shown in Fig. 1(b). The antenna array is 


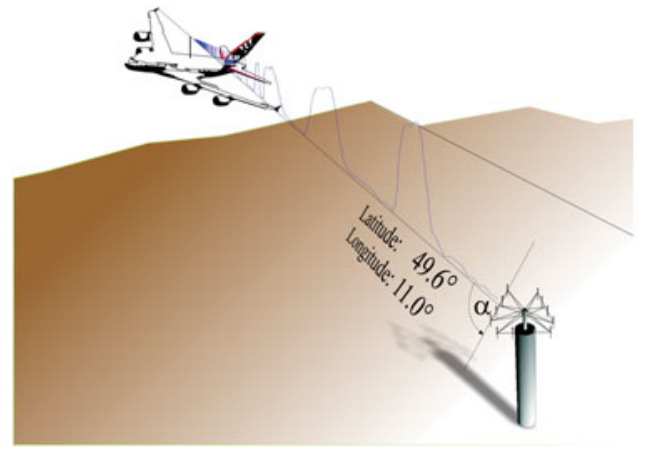

Verification of ADS-B position by a DOA sensor

(a)

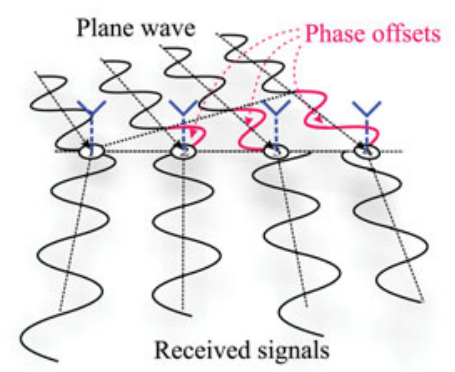

Principle of DOA estimation by a ULA

(b)

Fig. 1. ADS-B with DOA estimation.

restricted to a uniform linear array (ULA) with half wavelength spacing to avoid ambiguities. The signals received from the four antennas show a characteristic phase offset between neighboring channels according to the impinging angle. Ideally, this phase offset is equal between all neighboring channels.

If the receiving channels provide complex signal detection by the application of IQ mixers, the resulting phase properties in between the channels can be used to estimate the DOA of the impinging signal. The receivers include $A / D$ conversion to allow digital signal processing and the application of subspace-based DOA estimation.

The performance of multiple subspace-based DOA estimation approaches in SSR scenarios has been investigated in our previous work [5]. By the application of calibration methods based on eigenstructure analysis [6], the performances of all ESPRIT and MUSIC variants become very similar. It is thus sufficient to evaluate the results gathered by NC Unitary ESPRIT [7].

\section{ANTENNA ARRAY AND RECEIVER}

The antenna arrays used for the field tests are a halfwavelength spaced six-element monopole (see Fig. 2(b)) and an eight-element planar ULA (see Fig. 2(a)). Only the four inner elements of each array are used for reception, the remaining elements are terminated by $50 \Omega$ to homogenize the single antenna characteristics. Those passive elements are needed even in the case of eigenstructure-based calibration (as applied here) because of fringe effects due to the finite array length. The terminated antenna elements lower the parasitic phase and amplitude differences between the reception channels, and that way improve the direction of arrival estimation precision.

A larger number of terminated elements are thus always favorable. This becomes obvious by comparing the results gathered by both the eight-element planar and the six-element monopole array in the following section.

Each of the four inner antenna elements is connected to a dedicated analog receiver frontend, which converts the $1090 \mathrm{MHz}$ RF signal to an IF of $62 \mathrm{MHz}$. The resulting IF signals are band-pass filtered and fed to a four-channel, $50 \mathrm{MHz}, 8$-Bit digitizer card.

Digital signal processing such as IQ-demodulation and (matched) lowpass filtering is carried out in the digital domain on a PC. A quad-core processor and the concept of parallel computing are used to decode and process a maximum number of SSR telegrams.

The whole system is water-cooled, integrated in a weather resistant portable aluminum box and can be conveniently controlled by wireless LAN. A graphical user interface allows extensive sensor diagnoses for field testing, including a table of present aircraft, error histograms, and signal monitoring.

\section{MEASUREMENTS}

\section{A) Scenario}

In the following, two measurement campaigns are evaluated. One was conducted on the roof of a six-story university building, using ADS-B telegrams to compute a DOA reference. A total of 26000 ADS-B airborne position telegrams were recorded with each of the planar and the monopole array.

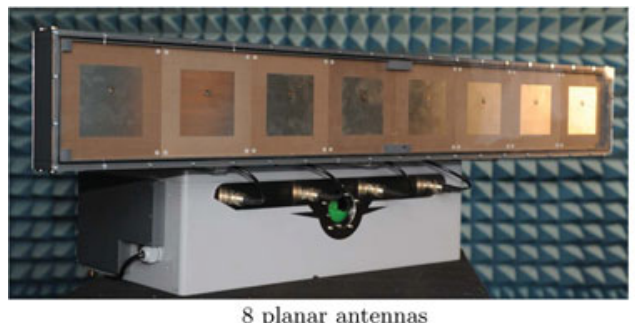

(a)

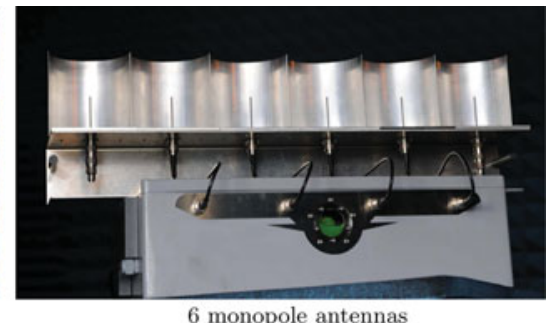

(b)

Fig. 2. Sensor equipped with two different antenna arrays. 
Airborne positions decoded from the ADS-B replies are plotted in Fig. 3(a), showing the angle and distance distribution of the positions relative to the DOA sensor. The position distribution is very similar to both the monopole and the planar array, so just the positions received by the planar array are shown. Due to the 8 - $\mathrm{Bit} \mathrm{A} / \mathrm{D}$-converter, the dynamic of the receiver is limited to around $48 \mathrm{~dB}$. Still transponders at a distance of up to 200 were received.

A small selected part of 33 telegrams (Airborne Position Code TYPE $\leq 13)$ is singled out and applied to calibrate the DOA sensor in the angular range from $45^{\circ}$ to $135^{\circ}$ based on the eigenstructure analysis.

The second measurement campaign was carried out at Adolf Würth Airport, Schwäbisch Hall, Germany. To evaluate the accuracy of the constructed DOA sensor, a dedicated reference aircraft (see Fig. 4(a)) is used. This measurement aircraft is owned and operated by the Institute of Flight Guidance, TU Braunschweig. It is equipped with a Novatel OEM IV receiver and an iMAR iVRU-FC Inertial Measurement Unit (IMU). In the first step of post processing, the recorded GPS data are improved by differential corrections. In a following step, the GPS data and the data provided by the inertial measurement unit are merged, taking the lever arms between GPS antenna and IMU into account. The resulting reference track provides accuracy in the sub-meter range.

The gathered reference positions shown in Fig. 3(b) are not ideally distributed over the angular range and the covered distance is smaller than the ADS-B campaign. The flight track consists mainly of several aerodrome circlings around the airport and then part of the aircraft track back to Braunschweig until connection is lost. The comparably small range is due to the low transponder power (7o W).

Nevertheless, 1170 Mode S telegrams were recorded and related to the aircraft position based on its time stamps. Again 33 telegrams were used for the eigenstructure calibration in the angular range from $45^{\circ}$ to $135^{\circ}$.

\section{B) Results}

First, an analysis of ADS-B reliability information is conducted. Therefore the database of ADS-B replies recorded by the planar antenna array is considered.

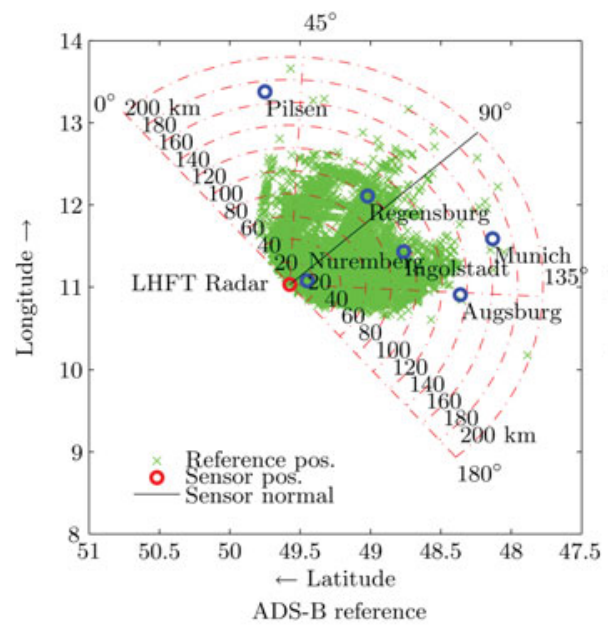

(a)
The telegrams in this database were transmitted by a large mixture of different transponders. Not all of those transponders provide reliable positioning information. The standard for ADS-B [8] defines a number of reliability classes encoded in the airborne position TYPE code. With each of those classes a dedicated containment radius around the transmitted position is defined in which the real position needs to be located with a percentage of $95 \%$ (compare Fig. 4(b)).

The database of recorded ADS-B telegrams contains TYPE $\in[10-14,17,18]$. The database is now sorted for TYPE-numbers and the RMSE of the differences between reference angles and DOA estimates is plotted versus the according TYPE in Fig. 5(a).

The RMSE remains about equal and below $1^{\circ}$ with all TYPE-numbers save TYPE $\in[17,18]$, where the RMSE ascends to more than $3^{\circ}$. As a correlation of low accuracy class and DOA RMSE is evident, those telegrams should not be trusted.

DOA error according to horizontal positioning error decreases with increasing distance, as can be anticipated from Fig. 4(b). The computed RMSE should therefore decrease over distance. Thus, the database is sorted for distance by bins of 30 and the resulting RMSE of each bin is plotted versus the corresponding distance in Fig. 5(b). As anticipated, a clear decrease of RMSE is observed with increasing distance when the whole database is regarded. If all telegrams with TYPE $>1$ (a total of 16000 telegrams remain) are excluded, the decrease in RMSE with increasing distance is much lower. At a distance of $135 \mathrm{~km}$ both curves meet, at an RMSE of $0.63^{\circ}$. It needs to be mentioned at this point that outliers have a heavy impact on this result due to the RMSE computation. A large number of ADS-B positions may be very accurate and just a low number of erroneous transponders suffices to spoil the overall result.

Using this selected database with TYPE $>13$, an error plot versus impinging angle can be computed as shown in Fig. 6(a). By restricting the angular range by $45^{\circ}$ to $135^{\circ}$, the histogram shown in Fig. 6(d) visualizes the according error distribution. The RMSE in this angular range computes to $0.9^{\circ}$. The same analysis was applied on the database recorded with the sixelement monopole antenna array. By comparing the results

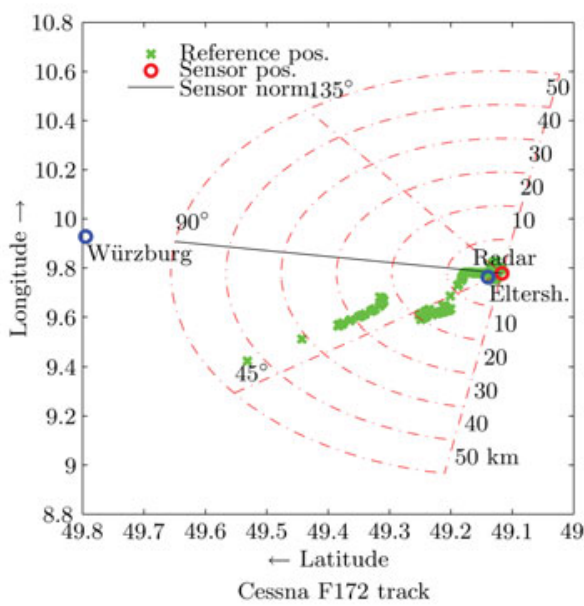

(b)

Fig. 3. Reference positions of the two measurement campaigns. 


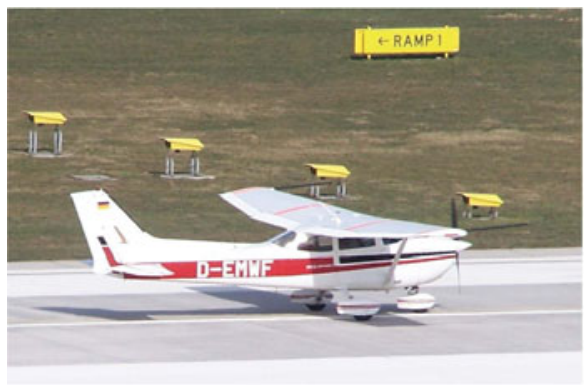

Measurement aircraft (TU Braunschweig): Cessna F172 Skyhawk

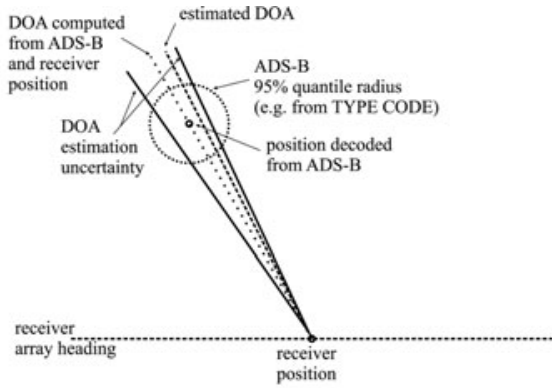

Example for a comparison of position decoded from ADS-B telegram and DOA estimation

(b)

Fig. 4. Measurement setup.

in Figs 6(b) and 6(e) with Figs 6(a) and 6(d), a larger error of the results gathered by the monopole array are obvious. As the performance by means of element cross coupling of both arrays is very similar, and subspace-based calibration is applied, this difference can be explained by the lower number of terminated elements. The resulting RMSE with the monopole array is $1.3^{\circ}$.

The results gathered by using the measurement aircraft and the receiver equipped with the planar array are shown in Figs 6(c) and 6(f). Regarding the RMSE values versus DOA, a slightly smaller error is observed. Computing the RMSE from $45^{\circ}$ to $135^{\circ}$ produces a value of $0.66^{\circ}$ by evaluating 616 reference positions in this range.

\section{APPLICATIONEXAMPLE: HUDSDNBAY}

With the DOA precision shown in the measurement results, pure DOA-based positioning will be very coarse in a wide area scenario. It thus might seem that multiangulation is restricted on small areas like airport approach or apron scenarios. In a wide area application, MLAT usually offers higher precision and requires lower receiver complexity (single channel receiver) of base station receivers. Still there are special scenarios, e.g. Hudson Bay, where DOA estimation can be advantageous. The area of surveillance is very large and can be approximated by a circle with a diameter of 900 .
As receivers can just be placed around the bay and their range is limited to around 400 by earth curvature, a MLAT system would require a large number of receivers and suffer from high dilution of precision due to improper geometry. Unfortunately the base station receivers would need to be placed in very remote areas where maintenance and access to electricity are expensive.

In comparison with MLAT, multiangulation requires just a minimum of two ground stations (instead of three with MLAT) within reach of the transponder for successful 2-D positioning. The positioning precision given by two DOA receivers in this scenario is computed as shown in [9] for Fig. 7 with a DOA sensor RMSE of $0.5^{\circ}$ and $0.1^{\circ}$. While the precision with the low accuracy sensors (Fig. $7(\mathrm{a})$ ) can be approximated between 2 and 6, the high accuracy sensors (Fig. 7 (b)) would lead to a positioning accuracy between 750 and 2. To ensure that at least two ground stations simultaneously receive a transponder anywhere over Hudson Bay, eight ground stations would be needed. Each of those eight ground stations would need to cover at least $180^{\circ}$. A homogeneous DOA estimation accuracy as shown in the results can only be realized withing $0^{\circ}$. One ground station would thus need to consist of two antenna arrays and multichannel receivers to get the accuracy shown in Fig. 7. The proposed multichannel receivers additionally introduce the possibility to apply spatial filtering to reduce interference. In the case of e.g. superimposed ADS-B telegrams, degarbling can be largely improved as shown in [10].

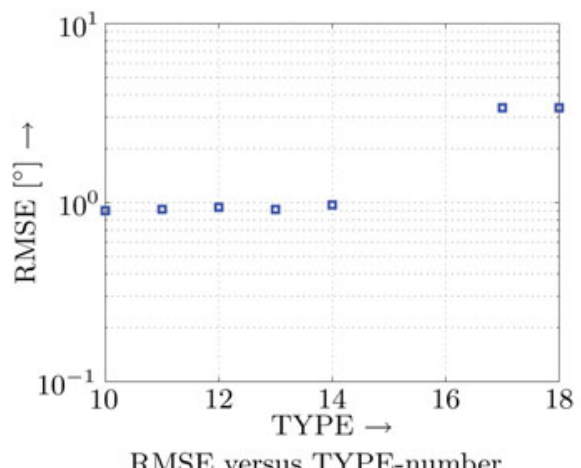

RMSE versus TYPE-number

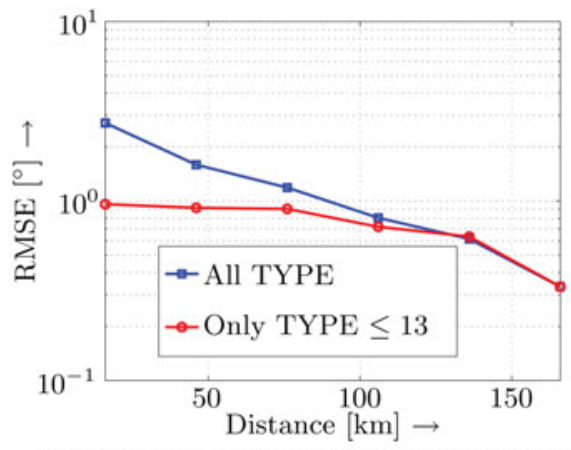

RMSE versus distance with and without TYPE-number filtering

Fig. 5. DOA estimation RMSE analysis. 


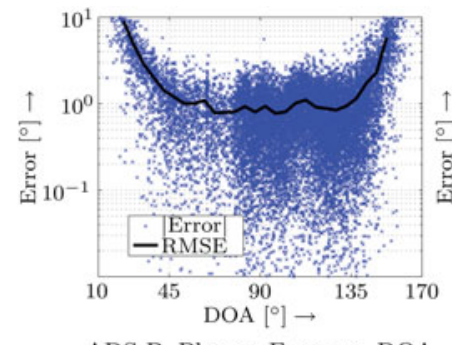

ADS-B, Planar: Error vs. DOA

(a)

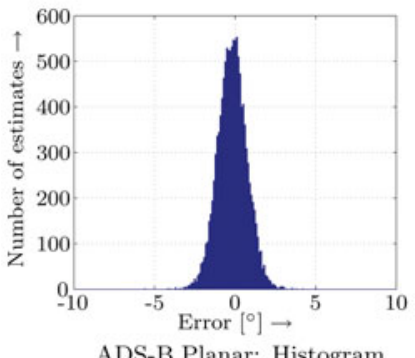

(d)

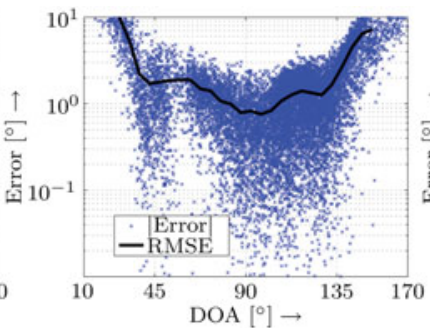

ADS-B, Monop.: Error vs. DOA

(b)

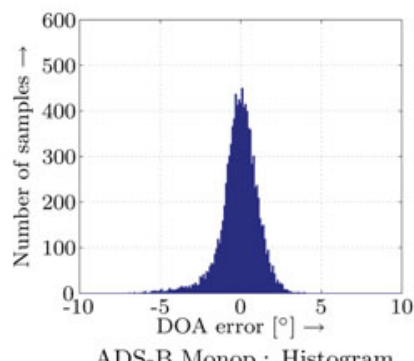

(e)

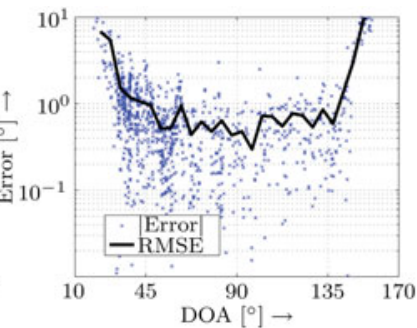

Ref. aircraft, Planar: Error vs. DOA

(c)

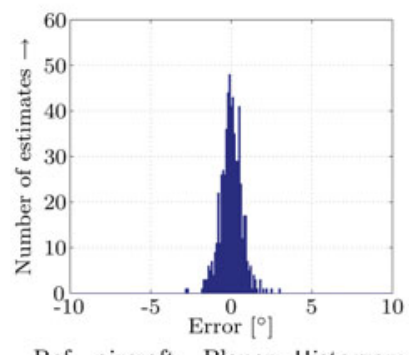

Ref. aircraft, Planar: Histogram

(f)

Fig. 6. DOA difference of ADS-B reference and DOA estimation sensor.

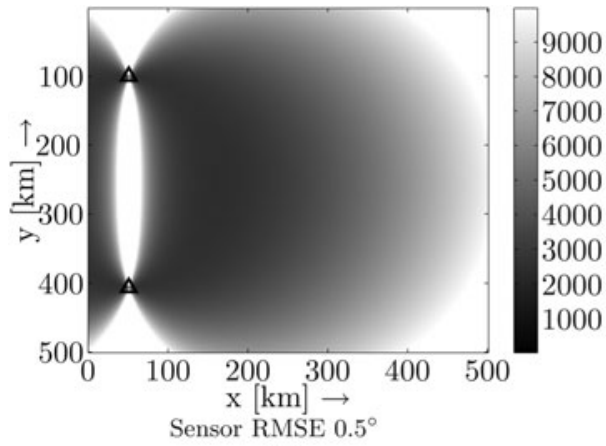

(a)

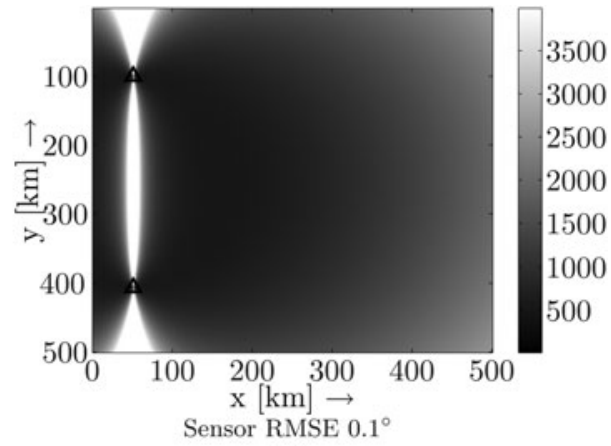

(b)

Fig. 7. Positioning precision of a 2 station multiangulation system.

Compared to 22 ground stations needed for an MLAT system as evaluated in [3], a multiangulation system seems more feasible.

\section{CONCLUSION}

To show the potential of ADS-B receivers with DOA estimation function, two measurement campaigns were conducted. By evaluating a large number of ADS-B airborne positions and comparing the resulting reference DOAs with estimated DOAs, a clear correlation between accuracy class and DOA error was found. Depending on the aircraft distance, a cross-check between DOA estimate and ADS-B position can thus gather valuable information regarding ADS-B reliability.

By a dedicated reference aircraft, the potential of the constructed DOA sensor to resolve DOA down to an RMSE of $0.66^{\circ}$ has been proven. Higher accuracies are theoretically possible. It has been shown that additional terminated antenna elements increase the DOA accuracy by homogenizing single antenna characteristics. Besides adding more antenna elements, dynamic increase by using a higher resolution $\mathrm{A} / \mathrm{D}$-converter would provide the means to increase estimation precision as well. Approaching DOA estimation accuracies in the range of $0.1^{\circ}$ will additionally need careful arrangement and sturdy construction of the antenna array. The application of averaging over multiple replies and tracking algorithms will be able to further increase the precision providing a suitable cross check for ADS-B.

\section{REFERENCES}

[1] Rekkas, C.; Rees, Melvyn: Towards ADS-B implementation in Europe, in ESAV'o8, 2008, pp. 7-4.

[2] Cedrini, V.; Zacchei, C.; Zampognaro, V.: ADS-B 1090ES implementation: the CRISTALMED project, in ESAV'o8, 2008, pp. 7-4.

[3] Barsheshat, Abraham A.: Implementation of ADS-B systems benefits and considerations, in Enhanced Surveillance of Aircraft and Vehicles Conference, 2011, pp. 7-4. 
[4] Reck, C.; Berold, U.; Schmidt, L.-P.: High precision DOA estimation of SSR transponder signals, in IEEE International Conference on Wireless Technology and Systems, 2010, pp. 7-4.

[5] Reck, C.; Berold, U.; Schmidt, L.-P.: Robust DOA estimation of SSR signals for aircraft positioning, in IEEE Radio Wireless Week, 2011, 8, pp. $73-76$

[6] Reck, C.; Berold, U.; Schuer, J.; Schmidt, L.-P.: Direction of arrival sensor calibration based on ADS-B airborne position telegrams, in European Radar Conference, 2009, pp. 77-80.

[7] Haardt, M.; Roemer, F.: Enhancements of unitary esprit for noncircular sources, in IEEE International Conference on Acoustics, Speech and Signal Processing, 2004, pp. 707-704.

[8] DO-260A: Minimum operational performance standards for $1090 \mathrm{MHz}$ extended squitter automatic dependent surveillance-broadcast (ADS-B) and traffic information services broadcast (TIS-B). Technical Report, RTCA, Inc., 2003.

[9] Levanon, N.: Lowest GDOP in 2-D scenarios. IEE Proc.-Radar, Sonar Navig., 147 (2000), 149-155.

[10] Reck, C.; Reuther, M.S.; Berold, U.; Schmidt, L.-P.: Spatial filtering and equalization for SSR signal detection in a multipath environment, in German Microwave Conference, 2011, pp. 7-4.

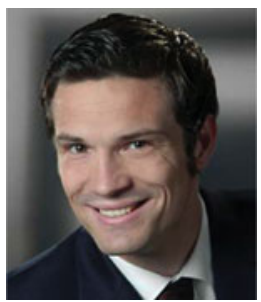

Christoph Reck was born 1981 in Forchheim, Germany. He received a Diploma degree in 2007 and a Ph.D. degree in 2011, both in Electrical Engineering from the University of Erlangen-Nuremberg. His research interests include multichannel receivers, smart antennas and direction finding techniques

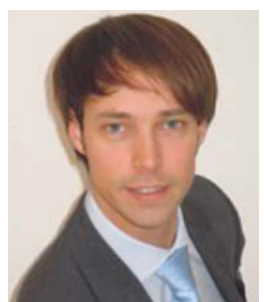

Max S. Reuther was born 1983 in Karlsruhe, Germany. He received his Bachelor of Science in General Engineering Science and his DiplomIngenieur in Electrical Engineering both from the Hamburg University of Technology in 2007 and 2009, respectively. In Hamburg, his main research focus was on antenna arrays at Ka-Band and Doherty power amplifiers at K-Band. Afterwards he spent a semester abroad at Sorbonne, Paris, studying French as well as French culture and history. In 2010, he then joined the Chair for High Frequency Technology at Friedrich-Alexander University, Erlangen, Germany, where he researched signal processing algorithms used in passive radar systems. Currently, he is an electrical development engineer at Rohde \& Schwarz, Munich, working in the area of spectrum analysis and EMI analyzers.

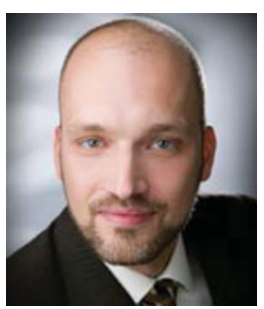

Alexander Jasch received his degree as Diplom-Ingenieur (certified engineer) from the Technische Universität Braunschweig in March 2008. He specialized in aerospace engineering. Since then he works as a research scientist at the Institute of Flight Guidance of the TU Braunschweig and is a member of the Institute's ATM department. His primary research interests are enhanced Mode-S-based localization techniques such as ADS-B and multilateration.

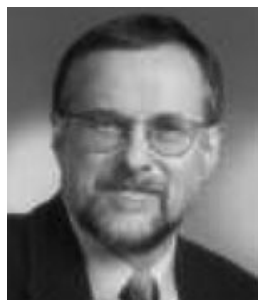

Lorenz-Peter Schmidt received the Diploma and Ph.D. degrees in electrical engineering from the Technical University of Aachen, Germany, in 1974 and 1979, respectively. From 1974 to 1979, he was with the Technical University of Aachen as a Research Assistant, dealing with the analysis of transverse discontinuities in microstrip lines. In 1979, he became a Post-Doctoral Research Associate with the University of Texas, Austin. From 1980 to 1998, he was with AEG-Telefunken, Ulm, Germany (later DASA, now CASSIDIAN), where he later became the Head of the Corporate Advanced Microwave and Millimeter-Wave Development Department. His R\&D activities included GaAs MMIC design up to $100 \mathrm{GHz}$, millimeter-wave interconnect and packaging technologies, radar sensors, transceiver front-end technology, and antennas in the range of $10-100 \mathrm{GHz}$. Since 1998, he has been a Full Professor and the Head of the Chair for Microwave Engineering and High-Frequency Technology at University of Erlangen-Nuremberg, Erlangen, Germany. His main research interests are in the fields of millimeter-wave and terahertz components and antenna technologies, as well as radar and active and passive imaging systems, laser technology, and microwave/photonic interaction. Dr Schmidt is a Member of VDE, the Chairman of the VDE/ITG Expert Group on Microwave Techniques, and a member of the German IEEE MTT/ AP Chapter Commission. In 2003, he served as the General Chairman of the European Microwave Week and the Chairman of the European Microwave Conference in Munich, and was the German representative in the EuMA General Assembly until 2010. 